\title{
A Circuit Theoretical Method for Efficient Finite Element Analysis of Acoustical Problems
}

\author{
A. Suat Ekinci, Abdullah Atalar \\ Department of Electrical and Electronics Enginecring, Bilkent University Bilkent, Ankara, 06533 \\ TURKEY
}

Abstract - In the last decade, there has been an outstanding improvement in the computer aided design tools for VLSI circuits regarding solution times and the circuit complexity. This study proposes formulating the acoustic field analysis problem using FEM, and employing the recent speed-up techniques used in the circuit simulators. In this work, total mass, stiffness and damping matrices are obtained using the FE approach, and piped into a computer program which generates an equivalent SPICE compatible circuit netlist. This approach makes it possible to use the most recent circuit simulation techniques to simulate the acoustical problems. The equivalent electrical circuit is a resistorinductor-capacitor (RLC) circuit containing controlled sources to handle the couplings. The circuit matrices are 6 times larger but are sparser. We analyze these circuits with a general-purpose circuit simulation program, HSPICE, which provides high accuracy solutions in a short time. We also use an in-house developed circuit simulation program, MAWE, which makes use of asymptotic waveform evaluation (AWE) technique that has been successfully used in circuit simulation for solutions of large sets of equations. The results obtained on several problems, which are solved in time and frequency domains using circuit simulators and the FE analysis program ANSYS, match each other pretty well. Using circuit simulators instead of conventional method improves simulation speed without a significant loss of accuracy.

\section{INTRODUC'IION}

Shrinking device sizes made it possible to build integrated circuits with large numbers of components. As a result of this advance, electrical circuit simulators that can handle large circuits have been developed. Those programs use new circuit simulation techniques that can quickly give highly accurate solutions. In this study, we obtain equivalent circuits and adopt the new techniques to acoustic field analysis problems.

Several studies have been done to use the simplicity of the circuit solution techniques in field analysis [1]. 'To couple finite element (FE) models with external circuits, the FE parts are solved separately and modeled as multi-port elements that communicate with the circuit model [2], [3].

In the recent years, electrical thermal network analogy is widely used to study the thermal behaviour of electronic components. In the work of Hsu, et. al. [4], elemental thermal circuit networks are developed and the obtained large resistor-capacitor (RC) networks are analyzed using a circuit simulator. Thermal problems give first order differential equations, and the algorithm may introduce negative valued resistors and capacitors.

In this study, we deal with second order differential equations, the algorithm we use, does not give negative values for circuit elements. Wo first apply the finite element formulation to the acoustic field problem to obtain the stiffness, mass and damping matrices. Then we find the circuit equivalents which will give the same solution. We analyze the obtained equivalent circuit using HSPICE (h97.2) [5] and in-housedeveloped MAWE [6].

\section{Finite Element formllation}

In a typical structural analysis, we try to find the stress and displacement distribution in a rigid body. under a set of loading and boundary conditions. In order to build a finite element (FE) model all the equations are written in terms of the displacement components and solved for unknown displacements [7].

After the necessary steps are completed, we obtain the following desired dymanic equations of motion of the structure which can be used to find frequency or time domain behaviour. or to find the matural frequencies and the mode shapes using eigcnvalue analysis techniques.

$$
\mathbf{M} \ddot{\mathbf{Q}}(t)+\mathbf{C} \dot{\vec{Q}}(t)+\mathbf{K} \overrightarrow{\mathbf{Q}}(t)=\overrightarrow{\mathbf{P}}(t)
$$

where $\mathbf{Q}(t)$ is the vector of nodal degrecs of freedom; i.e., the unknown vector, $M$ is the master mass matrix of the structure, $\mathbf{K}$ is the master stiffness matrix of the structure, and $\mathbf{C}$ is the master damping matrix of 
the structure. $\mathrm{C}$ is usually defined as

$$
\mathbf{C}=\alpha \mathbf{M}+\beta \mathbf{K}
$$

where $\alpha$ and $\beta$ are constants.

Once the vector $\overrightarrow{\mathbf{Q}}$ is found, the variations of the displacements, stresses and strains in the elements can be approximated.

\section{Circuit Simulation}

In modified node analysis (MNA) formulation linear circuits are defined by the equation:

$$
\mathbf{C} \dot{x}=-\mathbf{G} x+\mathbf{b} u(t)
$$

where $\mathbf{C}$ is the matrix of capacitances and inductors, $\mathbf{G}$ is the admittance matrix, $u(t)$ is the voltage or current excitation at the nodes defined by vector $\mathbf{b}$, and $x$ is the vector of nodal voltages, inductor currents and independent voltage supply currents.

The dynamic equation of motion (1) is of order 2; but it can be converted into an equation of order 1 [7], so that it can be solved using circuit simulation techniques.

\section{Moment Matching and Order Reduction}

The moment-matching techniques are widely used in rircuit simulation in order to reduce the execution time: [6]. [8]. [9] In these techniques by approximating the dominant poles of the circuit with a lower order morlel. the behaviour of the circuit is obtained.

Monem-matr hing uses the coefficients (moments) of the expansion of the system transfer function, $H(s)$, around a point in the complex s-plane. The Taylor scries expansion of $H(s)$ around $s_{0}$ is given as:

$$
H(s)=m_{0}+\left(s-s_{0}\right) m_{1}-\left(s-s_{0}\right)^{2} m_{2}+\ldots
$$

After the moments are generated, they are matched lo a ratio of two polynomials [6], [8], [10] or to a loworder set of poles and residues [9] by using Padé approximation.

The transfer function for a circuit which consists of lumped elements can be approximated as

$$
\hat{\mathbf{H}}(s)=\left(\mathbf{I}+\sigma \mathbf{A}+\sigma^{2} \mathbf{A}^{2}+\cdots+\sigma^{n} \mathbf{A}^{n}\right) \mathbf{r}
$$

where

$$
\begin{aligned}
& s=s_{o}+\sigma \\
& \mathbf{A}=-\left(\mathbf{G}+s_{o} \mathbf{C}\right)^{-1} \mathbf{C} \\
& \mathbf{r}=\left(\mathbf{G}+s_{o} \mathbf{C}\right)^{-1} \mathbf{b}
\end{aligned}
$$

As a result the moments are found using $\mathbf{m}_{n}=\mathbf{A}^{n} \mathbf{r}$. During the generation of moments the LU decomposition of the circuit matrix is calculated once for the first moment. Other moments are obtained using forward and backward substitutions.

After the moments are generated, the entries of the transfer function is approximated with a rational function.

$$
\hat{H}(s)=\frac{B(s)}{A(s)}=\frac{\sum_{i=0}^{p} b_{i} s^{i}}{\sum_{j=0}^{q} a_{j} s^{j}}
$$

In AWE, to approximate the behaviour of the circuit, the Taylor expansion around $s_{0}=0$ is evaluated. Since the information carried by the moments is accurate at low frequency region, the AWE technique is efficient in extracting the low frequency poles of the circuit. To improve the accuracy of AWE at relatively higher frequencies multi-point Padé approximation techniques were proposed $[6],[9],[10]$. In the work by Chiprout et. al. [9] complex frequency hopping $(\mathrm{CFH})$ technique is introduced. MAWE circuit simulation tool uses the method developed in the work of Celik et. al. [10]. MAWE provides good results also in high frequency regions as it uses the shifted moments, which provide the necessary information about the frequency range of interest. However, this approach requires the solution of the circuit matrix at several frequency points.

\section{EQUIVALENT CIRCUIT EXTRACTION}

There are many similarities in analyzing the systems of structures and circuits, we may solve the acoustic field analysis problems using a circuit simulator.

The first step is to change the second order differential equation (1) into a first order differential equation.

$$
\left[\begin{array}{c|c}
\mathbf{I} & \mathbf{0} \\
\hline \mathbf{0} & \mathbf{M}
\end{array}\right] \cdot \dot{\mathbf{y}}=-\cdot\left[\begin{array}{c|c}
\mathbf{0} & -\mathrm{I} \\
\hline \mathbf{K} & \mathbf{C}
\end{array}\right] \cdot \mathbf{y}+\left[\begin{array}{c}
\mathbf{0} \\
\mathbf{P}
\end{array}\right]
$$

where

$$
\mathbf{y}=\left[\begin{array}{c}
\overrightarrow{\mathbf{Q}}(t) \\
\dot{\overrightarrow{\mathbf{Q}}}(t)
\end{array}\right]
$$

We choose the unknowns as follows: displacements $(\overrightarrow{\mathbf{Q}}(t)) \rightarrow$ branch currents

$$
\text { velocities }(\dot{\overrightarrow{\mathbf{Q}}}(t)) \rightarrow \text { potential difference }
$$

between the nodes.

By looking at the entries of the system the circuit is constructed. The algorithm is of order $N^{2}$, and the time consumption is very small compared to the simulation time.

The circuit is realized using current through the inductors (L) the displacement, and the voltage difference between the inductor nodes as velocity. Currents 
through the capacitors are time derivatives of velocity. The other entries are simulated using current and voltage controlled current sources (CCCS and VCCS). All the circuit elements come in parallel so that no additional equations are needed, but to couple the currents one needs to add 0 volt voltage supplies (VS). In the circuit there are no negative valued capacitors and inductors, and no voltage source-inductor loops are introduced.

For each displacement-velocity pair, there are 6 rows in the circuit matrix, so its size is 6 times the original matrix sizes ( $M, C$ and $K$ ), but it is sparser. If the sparsity of the original matrices are $\alpha$ then the circuit matrix sparsity will be about $\frac{\alpha}{18}$.

\section{EXAMPLES}

In this section we will give some examples to demonstrate the speed of the method compared to the FEM solvers. The simulations are performed cn a SPARCstation 20 with $50 \mathrm{MHz}$ clock frequency by using FEM simulation program ANSYS (version 5.2 , electrical circuit simulation program HSPICE (h97.2), and in-house-developed circuit simulation tool MAWE.

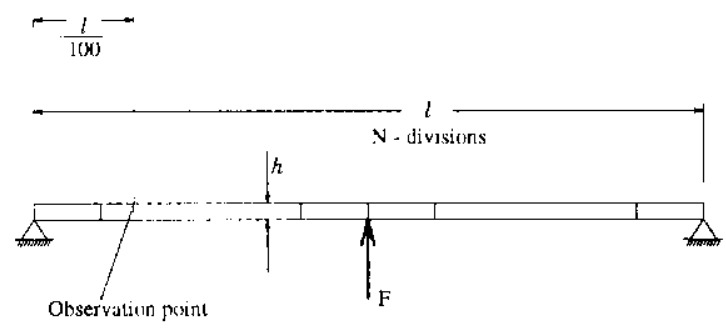

Fig. 1. Bar supported at the two sides (Example I).

\section{Example I}

First example is the harmonic analysis of a simple bar (Fig. 1). We have divided the bar into $\mathrm{N}$ elements, apply the force at the mid-point and observe the displacement at $1 / 100$ of the length. The system has $N-1$ UY unknowns (displacement in Y direction) and $N+1$ ROTZ unknowns (rotation around the z-axis), which gives a total of $2 N$ unknowns. The summary of extracted circuit is given in Table I. In Table II, times consumed (in seconds) during the harmonic analysis are shown. In AWE analysis 6th order Padé approximation is used, evaluation point is $s=0$. As the number of unknowns increase, the ratio of the execution times gets larger. It can be seen in Fig. 2

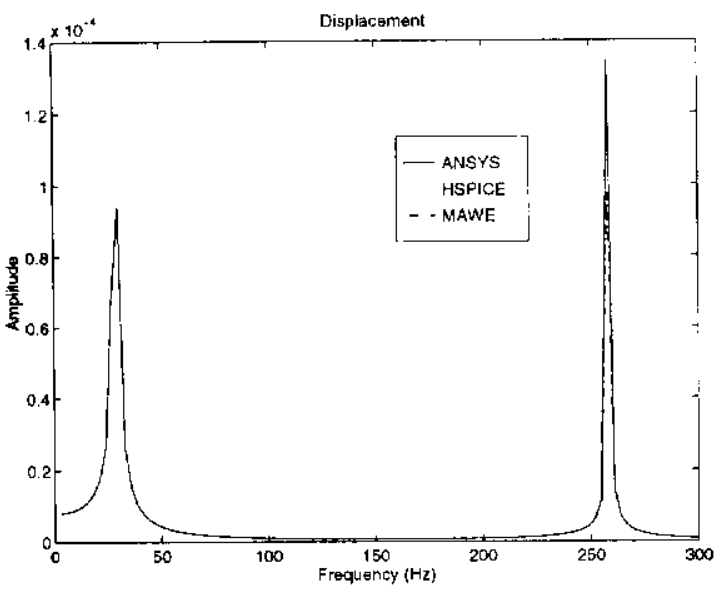

Fig. 2. Harmonic analysis results for Example I.

TABLE II

Timing Results for EXAMPLE I

\begin{tabular}{|c|r|r|r|}
\hline $\mathrm{N}$ & ANSYS & HSPICE & AWE \\
\hline \hline 100 & 57 & 2.28 & 1.83 \\
\hline 200 & 424 & 4.83 & 6.63 \\
\hline 400 & 4918 & 13.80 & 34.78 \\
\hline
\end{tabular}

that the harmonic response outputs are indistinguishable.

\section{Example II}

In this example, the transient behaviour of the mid point of the same bar, when a step function with finite rise time is applied to the same point(Fig. 1), is simulated. Approximation order is 6 ; and the matrix inversion is done only for $s=0$. In Table III the time consumptions (in seconds) are shown. It can be seen in Fig. 3 that the asymptotic waveform evaluation technique gives very good results in transient analysis.

TABLE III

TIMING RESULTS FOR EXAMPLE II

\begin{tabular}{|c|r|r|r|}
\hline $\mathrm{N}$ & \multicolumn{1}{|c|}{ ANSYS } & HSPICE & AWE \\
\hline \hline 100 & 8.11 & 8.55 & 1.84 \\
\hline 200 & 76.55 & 18.25 & 7.1 \\
\hline 400 & 686.01 & 39.25 & 35.3 \\
\hline
\end{tabular}

\section{CONCLUSION}

In this paper, we propose a method to convert the finite element formulations of the structural problems 
TABLE I

Circlit SUMmary For EXAMPLE I.

\begin{tabular}{|c|c||c|c|c|c|c|c|}
\hline \multicolumn{2}{|c||}{ FE Problem } & \multicolumn{5}{c|}{ Electrical Circuit } \\
\hline Unknowns & matrix size & L & C & CCCS & VS & Circuit nodes & circuit matrix size \\
\hline 200 & 200 & 200 & 200 & 1788 & 400 & 601 & 1201 \\
400 & 400 & 400 & 400 & 3628 & 800 & 1201 & 2401 \\
800 & 800 & 800 & 800 & 7138 & 1600 & 2401 & 4801 \\
\hline
\end{tabular}

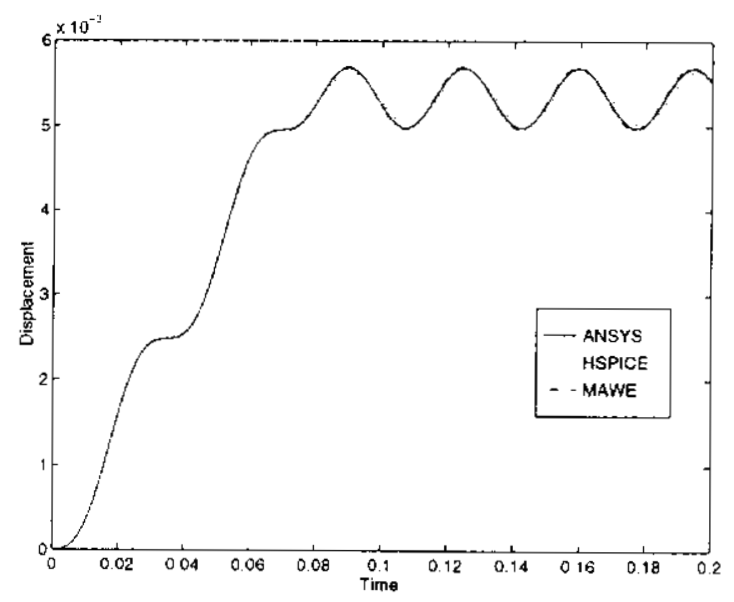

Fig. 3. Transient analysis results of the three methods for Example II.

into circuit analysis problems so that they can be solved with a general purpose circuit simulator.

Equivalent circuit extraction is done by a computer program which takes the total mass and stiffness matrices as input and creates a circuit netlist file. The resultant circuit matrix is 6 times larger. In the solution of this equivalent circuit, instead of solving the system of equations at each frequency point momentmatching techniques can be used. The main reason behind the efficiency of this method is the decrease in the number of LU decompositions. Multi-point AWE technique requires one LU decomposition per expansion point. Generally, the number of expansion points is less than the number of frequency points to get an accurate solution.

Several examples have been studied using the proposed method and an accurate match with the finite element method results has been obtained. Without a significait loss of accuracy, the simulation speed is improved using AWE. With our in house developed sparse matrix solver, which is not a particularly fast one, this potential improvement with respect to HSPICE program cannot be seen in the figures. With a better sparse matrix sparse matrix solver, the expected speed-up ratio is almost equal to the number of frequency points divided by the number of expansion points.

\section{REFERENCES}

[1] Gabriel Kron, "Equivalent circuits of the elastic field," Journal of Applied Mechanics, vol. 11, pp. A149-A161, September 1944

[2] Janne Väänänen, "Circuit theoretical approach to couple two-dimensional finite element models with external circuit equations," IEEE Transactions on Magnetics, vol. 32, no. 2, pp. 400-410, March 1996.

[3] Thomas E. McDermott, Ping Zhou and John Gilmore, "Electromechanical system simulation with models generated from finite element solutions," IEEE Transactions on Magnetics, vol. 33, no. 2, pp. 1682-1685, March 1997.

[4] Jia Tzer Hsu and Loc Vu-Quoc. "A rational formulation of thermal circuit models for electrothermal simulation . part I: Finite element method," IEEE Transactions on Circuits and Systems-1: Fundametal Theory and Applications, vol. 43, no. 9, pp. 721-732, September 1996.

[5] META-SOFTWARE, Canpbell, CA, HSPICE User's Manual, 1996.

[6] M. Sungur, A.S. Ekinci and A. Atalar, "An efficient program for analysis of interconnect circuits," International Journal of Electronicis, vol. 42, pp. 6-13, 1997.

[7] S.S. Rao, The Finite Element Method in Engineering, Pergamon Press, Oxford, 2nd edition, 1989.

[8] L. T. Pillage and R. A. Rohrer, "Asymptotic waveform evaluation for timing analysis," IEEE Transactions on Computer Aided Design of Integrated Circuits and Systems, vol. 9, pp. 352-366, April 1990.

[9] E. Chiprout and M. S. Nakhla, "Analysis of interconnect networks using complex frequency hopping $(\mathrm{CFH})$ :" IEEE Transactions on Computer Aided Design of Integrated Circuits and Systems, vol. 14, no. 2, pp. 186-200, February 1995.

[10] M. Celik, O. Ocali, M.A. Tan and A. Atalar, "Polezero computation in microwave circuits using multi point pade approximation," IEEE Transactions on circuits and systems-I: Fundamental Theory and Applications, vol. 42, pp. $6-13,1995$. 